\title{
LETTERS
}

\section{Opioid agonist dosage adequacy from clinical and patient perspectives: further considerations}

We read with great interest the CMAJ article by Artenie and colleagues ${ }^{1}$ showing that the risk of hepatitis $\mathrm{C}$ virus ( $\mathrm{HCV}$ ) infection among people who inject drugs varies considerably depending on the dosage of opioid agonist treatment and patient perception of their dosage. We applaud the authors for their excellent study, which makes a valuable contribution to the literature. However, we would like to point out a potential, clinically relevant methodologic limitation of that study. In addition, we wish to highlight one of the findings to contextualize better the overall results of the study.

Based on clinical practice guidelines, Artenie and colleagues ${ }^{1}$ categorized the prescribed dosages of methadone and buprenorphine as high when they were $60 \mathrm{mg}$ or more per day and $16 \mathrm{mg}$ or more per day, respectively. Dosages under those cut-offs were considered low. However, the effective dosage can range widely among individuals, and thus the dichotomous cut-offs used in that study may be less than optimal. ${ }^{2}$

Moreover, the use of individualized, flexible dosing strategies is supported by robust evidence. ${ }^{3}$ Studies have shown that this individualized treatment approach should not focus on the dosage level but on dosage adequacy (from both clinical and patient perspectives). ${ }^{4}$ In this regard, the clinical adequacy of the dosage can be evaluated using the Opiate Dosage Adequacy Scale (ODAS), a psychometrically sound tool that has parallel versions for methadone ${ }^{5}$ and buprenorphine. ${ }^{6}$

Had the ODAS (or a similar instrument) been used in the study by Artenie and colleagues, ${ }^{1}$ the number of patients in the exposure categories derived from the 2-by-2 table of dosage adequacy (clinician v. patient perspectives) would likely have been different.

Our comments are not intended to detract from the importance of this study, which provides a valuable contribution to the growing body of evidence showing that patient-perceived dosage adequacy is a critical factor in the effectiveness of opioid agonist maintenance treatment. This finding is particularly relevant given that patient perceptions of dosage adequacy may be an indicator of the level of implementation of shared decision-making processes in opioid agonist maintenance treatment, ${ }^{7}$ an essential characteristic of genuinely patient-centred care for people with opioid use disorder.

\section{Joan Trujols PhD}

Clinical psychologist, Unitat de Conductes Addictives, Servei de Psiquiatria, Hospital de la Santa Creu i Sant Pau, Barcelona, Spain

\section{Josefa Campins MSc}

Clinical psychologist, Unitat de Conductes Addictives, Servei de Psiquiatria, Hospital de la Santa Creu i Sant Pau, Barcelona, Spain

\section{Elisa Ribalta MD}

Psychiatrist, Unitat de Conductes Addictives, Servei de Psiquiatria, Hospital de la Santa Creu i Sant Pau, Barcelona, Spain

- Cite as: CMAJ 2019 September 30; 191:E1084. doi: 10.1503/cmaj.73048

\section{References}

1. Artenie AA, Minoyan N, Jacka B, et al. Opioid agonist treatment dosage and patient-perceived dosage adequacy, and risk of hepatitis $\mathrm{C}$ infection among people who inject drugs. CMAJ 2019;191:E462-8.

2. Trafton JA, Minkel J, Humphreys K. Determining effective methadone doses for individual opioiddependent patients. PLoS Med 2006;3:e80.

3. Bao YP, Liu ZM, Epstein DH, et al. A meta-analysis of retention in methadone maintenance by dose and dosing strategy. Am J Drug Alcohol Abuse 2009;35:28-33.

4. Roux P, Lions C, Michel L, et al.; ANRS Methaville Study Group. Predictors of non-adherence to methadone maintenance treatment in opioiddependent individuals: implications for clinicians. Curr Pharm Des 2014;20:4097-105.

5. González-Saiz F, Lozano Rojas O, Ballesta Gómez R, et al.; Serum Methadone Levels Study Group. Evidence of reliability and validity of the Opiate Dosage Adequacy Scale (O.D.A.S.) in a sample of methadone maintenance patients. Heroin Addict Relat Clin Probl 2008;10:25-38.

6. González-Saiz F, Lozano Rojas O, Trujols J, et al. Evidence of validity and reliability of the Opiate Dosage Adequacy Scale (ODAS) in a sample of heroin addicted patients in buprenorphine/naloxone maintenance treatment. Drug Alcohol Depend 2018;183:127-33.

7. Trujols J, González-Saiz F, Manresa MJ, et al. Patient perception of methadone dose adequacy in methadone maintenance treatment: the role of perceived participation in dosage decisions. Patient Educ Couns 2017;100:981-6.

Competing interests: None declared. 